\title{
Advanced glycation end products are associated with arterial stiffness in type 1 diabetes
}

\author{
Gemma Llauradó ${ }^{1,5}$, Victòria Ceperuelo-Mallafré ${ }^{2}$, Carme Vilardell ${ }^{3}$, Rafael Simó ${ }^{4}$, \\ Pilar Gil ${ }^{5}$, Albert Cano', Joan Vendrell ${ }^{5}$ and José-Miguel González-Clemente ${ }^{1}$ \\ ${ }^{1}$ Department of Diabetes, Endocrinology and Nutrition, Hospital de Sabadell, Corporació Sanitària i Universitària \\ Parc Taulí (Universitat Autònoma de Barcelona), Parc Taul s/n, 08208 Sabadell, Spain \\ ${ }^{2}$ Centro de Investigación Biomédica en Red-Fisiopatología de la Obesidad y la Nutrición (CIBERobn), Hospital Clínico \\ Virgen de la Victoria, Málaga, Spain \\ ${ }^{3}$ Diabetes, Endocrinology and Nutrition Unit, Hospital Sant Joan de Déu de Manresa, Xarxa Assistencial Althaia, \\ Manresa, Spain \\ ${ }^{4}$ Centro de Investigación Biomédica en Red de Diabetes y Enfermedades Metabólicas Asociadas (CIBERDEM) \\ and Diabetes and Metabolism Research Unit, Institut de Recerca Hospital Universitari Vall d'Hebrón (VHIR), \\ Universitat Autònoma de Barcelona, Barcelona, Spain \\ ${ }^{5}$ Centro de Investigación Biomédica en Red de Diabetes y Enfermedades Metabólicas Asociadas (CIBERDEM), \\ Hospital Universitari Joan XXIII de Tarragona, Institut d'Investigació Sanitària Pere Virgili, Universitat Rovira i Virgili, \\ Tarragona, Spain
}

Correspondence should be addressed to J-M González-Clemente Email josmi.gonza@gmail.com

\begin{abstract}
The aim of this study was to investigate the relationship between advanced glycation end products (AGEs) and arterial stiffness (AS) in subjects with type 1 diabetes without clinical cardiovascular events. A set of 68 patients with type 1 diabetes and 68 age- and sex-matched healthy subjects were evaluated. AGEs were assessed using serum concentrations of $\mathrm{N}$-carboxymethyl-lysine (CML) and using skin autofluorescence. AS was assessed by aortic pulse wave velocity (aPWV), using applanation tonometry. Patients with type 1 diabetes had higher serum concentrations of CML (1.18 vs $0.96 \mu \mathrm{g} / \mathrm{ml} ; P=0.008)$ and higher levels of skin autofluorescence (2.10 vs 1.70; $P<0.001$ ) compared with controls. These differences remained significant after adjustment for classical cardiovascular risk factors. Skin autofluorescence was positively associated with aPWV in type 1 diabetes $(r=0.370 ; P=0.003)$. No association was found between $\mathrm{CML}$ and aPWV. Skin autofluorescence was independently and significantly associated with aPWV in subjects with type 1 diabetes $(\beta=0.380 ; P<0.001)$ after adjustment for classical cardiovascular risk factors. Additional adjustments for $\mathrm{HbA} 1 \mathrm{c}$, disease duration, and low-grade inflammation did not change these results. In conclusion, skin accumulation of autofluorescent AGEs is associated with AS in subjects with type 1 diabetes and no previous cardiovascular events. These findings indicate that determination of tissue AGE accumulation may be a useful marker for AS in type 1 diabetes.
\end{abstract}

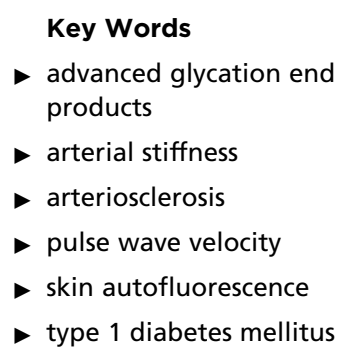

Journal of Endocrinology (2014) 221, 405-413

\section{Introduction}

Cardiovascular disease is the major cause of mortality in type 1 diabetes (Libby et al. 2005). Diabetes mellitus results in an accelerated arteriosclerotic process, which is not fully explained by classical cardiovascular risk factors. As a result, the pathophysiological mechanisms underlying cardiovascular events in type 1 diabetes are not completely understood. 
Arterial stiffness (AS) is an early sign of arteriosclerosis (Cavalcante et al. 2011) and predicts cardiovascular events independently of classical cardiovascular risk factors in several populations (Vlachopoulos et al. 2010). The exact mechanisms responsible for the increase in AS are not fully understood, but are likely to reflect a complex interaction between structural and functional changes in the arterial wall (Zieman et al. 2005). The structural changes are characterised by an overproduction of abnormal collagen and diminished quantities of normal elastin induced by an inflammatory milieu (Zieman et al. 2005). In this context, we have recently shown that low-grade inflammation is independently associated with an increase in AS in type 1 diabetes (Llaurado et al. 2012). However, other mechanisms, such as the formation of advanced glycation end products (AGEs), are also thought to be involved in the increase in AS that occurs in diabetes (Zieman et al. 2005).

AGEs are the result of non-enzymatic irreversible glycation and oxidation of proteins, lipids and nucleic acids. Hyperglycaemia and oxidative stress accelerate the accumulation of AGEs (Singh et al. 2001), which are involved in the development of micro- and macrovascular complications in type 1 diabetes (Genuth et al. 2005, Nin et al. 2011). AGEs can be assessed by chemical characterisation: pentosidine and $\mathrm{N}$-carboxy-methyl-lysine (CML) are some examples of well-characterised AGEs. Interestingly, certain dermal AGEs exhibit autofluorescent properties and the measurement of skin autofluorescence has been proposed as a novel marker of tissue accumulation of AGEs (Meerwaldt et al. 2004).

The gold standard for measuring central AS is aortic pulse wave velocity (aPWV). Higher aPWV is reflective of AS. AGEs have been associated with an increase in aPWV in healthy individuals (Semba et al. 2009), hypertensive individuals (McNulty et al. 2007), subjects with end-stage renal failure (Ueno et al. 2008) and patients with type 2 diabetes (Choi et al. 2009). In type 1 diabetes, AGEs have been associated with an increase in pulse pressure, a surrogate marker for central AS (Schram et al. 2005). However, whether a relationship exists between AGEs and AS measured by aPWV in type 1 diabetic patients without previous clinical cardiovascular events (coronary heart disease, cerebrovascular disease or peripheral vascular disease) is not known.

The main aim of the present study was to test the strength of the relationship between two different measurements of AGEs (skin autofluorescence and serum levels of $\mathrm{CML}$ ) and aPWV (as a surrogate for central AS) in patients with type 1 diabetes without clinical cardiovascular events.

\section{Subjects and methods}

\section{Subjects}

A set of 68 patients with type 1 diabetes, aged 18-65 years, and 68 age- and sex-matched healthy subjects were included in our study. None of the subjects had any clinical cardiovascular disease. Also, none had any condition associated with an inflammatory response (e.g. acute or chronic infectious disease) or had received antiinflammatory treatment in the previous 6 months. Subjects with type 1 diabetes were consecutively recruited from our outpatient clinic and all had at least 1 year of evolution.

\section{Clinical study}

The study methods have previously been described in detail elsewhere (Llaurado et al. 2012). Briefly, after an overnight fast, venous blood samples were taken and aliquots of plasma and serum were stored at $-80^{\circ} \mathrm{C}$ until processed. In women, all measurements were taken during the follicular phase of the menstrual cycle. The following information was recorded using a predefined standardised form: sex, age, diabetes duration, BMI, waist-to-hip ratio (WHR), systolic and diastolic blood pressure (SBP and DBP), and mean arterial pressure (MAP) - defined as 1/3 SBP + 2/3 DBP - physical activity (International Physical Activity Questionnaire (IPAQ) (Hallal \& Victora 2004)), cigarette smoking, alcohol intake, insulin dose or any other drug treatment, and microvascular complications (assessed only in patients with type 1 diabetes as previously described (Llaurado et al. 2012)).

Hypertension was defined as having a blood pressure of over 140/90 (Mancia et al. 2007) and/or being under antihypertensive treatment. Dyslipidaemia was defined as having a total cholesterol concentration of over $5.2 \mathrm{mmol} / \mathrm{l}$, triglycerides over $1.7 \mathrm{mmol} / \mathrm{l}$, HDL-cholesterol of less than $1.03 \mathrm{mmol} / \mathrm{l}$, LDL-cholesterol of over $3.4 \mathrm{mmol} / \mathrm{l}$ (NCEP 2002) and/or receiving drug treatment for dyslipidaemia.

The study protocol was approved by our hospital's Ethics Committee (Sabadell Hospital Clinical Research Ethics Committee) and conducted according to the principles of the Declaration of Helsinki. All subjects gave their written informed consent before participating in the study.

\section{Laboratory analyses}

HbA1c was determined by HPLC (Menarini Diagnostics, Firenze, Italy). Total serum cholesterol, triglycerides,

Published by Bioscientifica Ltd 
HDL-cholesterol and LDL-cholesterol were measured using standard enzymatic methods.

AGEs can act via their receptor (RAGE) triggering multiple signalling cascades, resulting in the activation of nuclear transcription factors and the secretion of proinflammatory cytokines and vascular adhesion molecules (Goldin et al. 2006). To evaluate the relationship between the three mechanisms, the main markers of low-grade inflammation, endothelial dysfunction (focusing on those markers implicated in cellular adhesion and permeability) and AGEs were evaluated. High-sensitivity C-reactive protein (hsCRP) was determined by immunonephelometry (Siemens, Munich, Germany). ELISA was used for the assessment of interleukin 6 (IL6; R\&D Systems, Abingdon, UK), soluble fractions of tumor necrosis factor $\alpha$ receptors 1 (sTNF $\alpha$ R1; Hycultbiotech, Uden, The Netherlands) and 2 (sTNF $\alpha$ R2; R\&D Systems), soluble intercellular adhesion molecule 1 (ICAM1; Boster Biological Technology, Wuhan, China), soluble vascular cell adhesion molecule 1 (VCAM1; Boster Biological Technology) and E-selectin (Boster Biological Technology). ELISA was used also for the assessment of CML (Cylex Co. Ltd, Nagano, Japan; Komosinska-Vassev et al. 2012). The test was carried out according to the manufacturer's instructions. The mean minimum detectable concentration was $0.126 \mathrm{ng} / \mathrm{ml}$. The antibodies in the CML kit are highly specific for CML-adducts, with no detectable cross reactivity to non-CML proteins that may be present in human serum. The intra- and inter-assay coefficients of variation (CV) were 5.2-7.4 and 4.7-15.2\% respectively for CML.

\section{Skin autofluorescence}

Skin autofluorescence was assessed using an AGE Reader (DiagnOptics BV, Groningen, The Netherlands). Three measurements per patient were taken at room temperature on the forearm, in three different positions at around $5-10 \mathrm{~cm}$ below the elbow fold, with patients in a sitting position. Briefly, the AGE Reader illuminates approximately $1 \mathrm{~cm}^{2}$ of skin surface (guarded against surrounding light), with an excitation light source of 300-420 nm. The light from the skin was measured using a spectrometer (model PC-1000 fiber optic spectrometer, Ocean Optics, Dunedin, FL, USA) in the range of $300-600 \mathrm{~nm}$, using $200 \mu \mathrm{m}$ glass fibre (Farnell, Leeds, UK). Autofluorescence is calculated as the average light intensity per nanometer in the range between 420 and $600 \mathrm{~nm}$, divided by the average light intensity per nanometer in the range between 300 and $420 \mathrm{~nm}$ (autofluorescence), multiplied by 100 and expressed as arbitrary units (AU). This technique has been previously validated for both type 1 and 2 diabetes (Meerwaldt et al. 2004). The CV for the three-repeated measurement was $4.2 \%$.

\section{Assessment of AS}

Measurement of aPWV Subjects rested in supine position and measurements were taken in accordance with the recommendations of the recent consensus on AS (Laurent et al. 2006). The method has been previously described elsewhere in detail (Llaurado et al. 2012). Briefly, aPWV was determined by sequential applanation tonometry (Millar tonometer: SPC-301; Millar Instruments, Houston, TX, USA) at the carotid and femoral arteries, gated to a three-lead ECG, was recorded using the SphygmoCor device (SphygmoCor; AtCor, Sydney, Australia). Those aPWV not fulfilling the automatic quality controls specified by the SphygmoCor software were rejected. The mean of two aPWV measurements was taken for each subject for all calculations. The CV for the two repeated measurements was $3.11 \%$. Data were available for all participants included in the study.

\section{Statistical analyses}

Data are presented as percentages, mean (s.D.) for normally distributed variables, and median (interquartile range) for non-normally distributed variables. All data were tested for normality using the Kolmogorov-Smirnov test. Differences between patients with type 1 diabetes and control patients were analysed using the $\chi^{2}$-test for comparisons of proportions and unpaired $t$-tests or Mann-Whitney $U$ tests for comparisons of quantitative variables as needed. Although patients were recruited to be age- and sex-matched, we performed the analyses using an independent $t$-test. We chose this strategy because it was statistically more conservative (although it is less likely to detect differences between groups). We assessed the potential relationships between all variables through univariate Spearman correlations. To assess the relationship between AGEs and AS, linear regression models were used to adjust for potential confounders, using aPWV as the dependent variable. Independent variables for linear regression analyses were selected based on univariate correlation analyses and those variables known or likely to be associated with AGEs or AS. To improve skewness and kurtosis, and to improve linearity, non-normally distributed variables were log-transformed. Collinearity tests (tolerance and the reciprocal of tolerance: the variance inflation factor) were performed first to avoid

Published by Bioscientifica Ltd 
overlap between introduced variables (threshold for the tolerance of VIF $>10$ ). Because we measured inflammatory-related serum proteins and endothelial dysfunction markers only once, the association (if any) with AGEs and AS would tend to be underestimated. To address this issue, a $Z$-Score was calculated for each marker as follows: (value for the individual-mean value for the study population)/s.D. Subsequently, the low-grade inflammation general score was calculated as ( $Z$-score for $\mathrm{hsCRP}+Z$-score for IL6 $+Z$-score for sTNF $\alpha \mathrm{R} 1+Z$-score for sTNF $\alpha$ R2)/4 and an endothelial dysfunction general score was calculated as $(Z$-score for ICAM $1+Z$-score for VCAM $1+Z$-score for E-selectin)/3. The IBM SPSS Statistics package (v. 19 for Mac; SPSS, Inc., IBM Company) was used for all calculations. All $P$ values were two-sided and a $P$ value of $<0.05$ was considered statistically significant.

Table 1 Clinical characteristics of study population

\begin{tabular}{|c|}
\hline 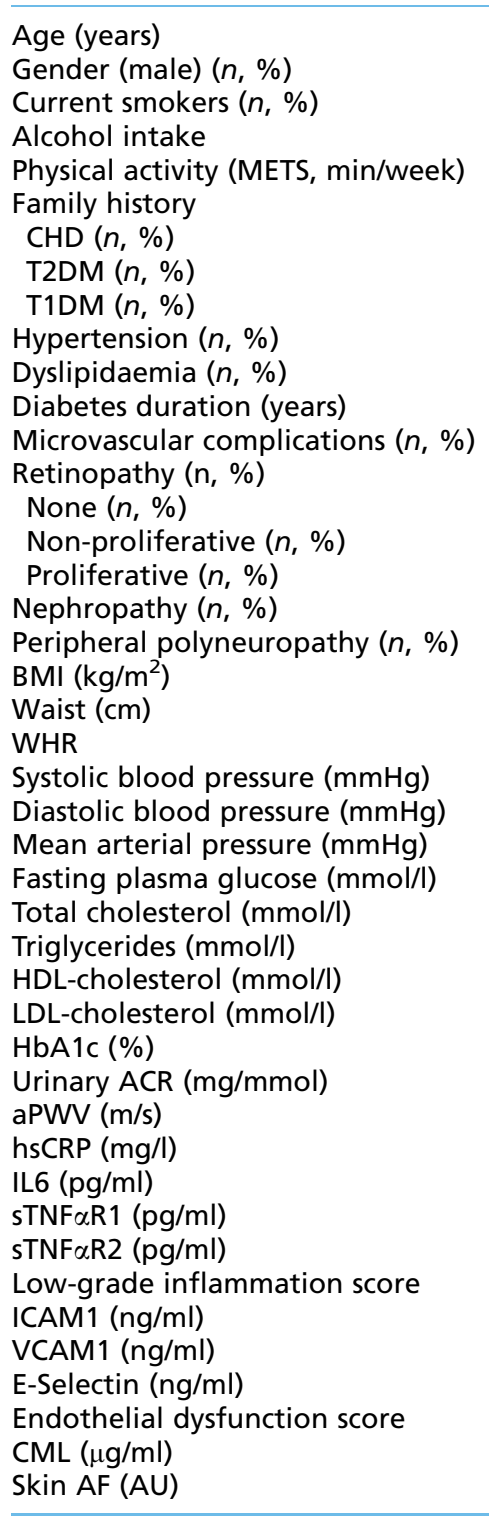 \\
\hline
\end{tabular}

\begin{tabular}{|c|}
\hline Healthy subjects $(n=68)$ \\
\hline $35.4(10.2)$ \\
\hline $34(50)$ \\
\hline $16(23.5)$ \\
\hline $1.43(0.00$ to 5.36$)$ \\
\hline 1386.0 (784.5 to 2079.0 ) \\
\hline $6(8.8)$ \\
\hline $12(17.6)$ \\
\hline $1(1.5)$ \\
\hline $3(4.4)$ \\
\hline $34(50)$ \\
\hline- \\
\hline- \\
\hline- \\
\hline- \\
\hline- \\
\hline- \\
\hline- \\
\hline- \\
\hline $24.0(3.1)$ \\
\hline $83.7(11.3)$ \\
\hline $0.85(0.1)$ \\
\hline $120.6(10.4)$ \\
\hline $70.8(8.4)$ \\
\hline $87.4(8.6)$ \\
\hline $4.67(0.53)$ \\
\hline $5.16(1.33)$ \\
\hline 0.80 (0.62 to 1.17$)$ \\
\hline 1.50 (1.19 to 1.87$)$ \\
\hline 2.81 (2.25 to 3.58$)$ \\
\hline $5.3(5.2$ to 5.5$)$ \\
\hline 0.39 (0.28 to 0.57$)$ \\
\hline $6.1(5.5$ to 6.7$)$ \\
\hline $0.8(0.4$ to 1.5$)$ \\
\hline $0.3(0.2$ to 0.5$)$ \\
\hline 1535.1 (1190.0 to 2845.6$)$ \\
\hline 2201.8 (1880.2 to 2561.5$)$ \\
\hline$-0.35(-0.63$ to 0.16$)$ \\
\hline 55.1 (48.3 to 62.7$)$ \\
\hline 715.8 (541.2 to 954.7$)$ \\
\hline 73.6 (50.9 to 99.1$)$ \\
\hline$-0.28(-0.58$ to 0.02$)$ \\
\hline $0.96(0.77$ to 1.23$)$ \\
\hline $1.7(1.6$ to 2.1$)$ \\
\hline
\end{tabular}

\begin{tabular}{c}
\hline Type 1 diabetes $(n=68)$ \\
\hline $35.3(10.1)$ \\
$34(50)$ \\
$24(35.3)$ \\
$1.43(0.00$ to 5.71$)$ \\
$1416.0(713.3$ to 2367.0$)$ \\
$3(4.4)$ \\
$16(23.5)$ \\
$5(7.4)$ \\
$17(25.0)$ \\
$32(47.1)$ \\
$13.0(7.3$ to 19.0$)$ \\
$16(23.5)$ \\
$10(14.7)$ \\
$58(85.3)$ \\
$6(8.8)$ \\
$4(5.9)$ \\
$9(13.2)$ \\
$0(0)$ \\
$25.7(3.6)$ \\
$85.3(12.0)$ \\
$0.86(0.1)$ \\
$125.0(12.1)$ \\
$72.9(8.3)$ \\
$90.3(8.7)$ \\
$9.15(3.66)$ \\
$4.80(0.87)$ \\
$0.78(0.61$ to 0.97$)$ \\
$1.70(1.21$ to 1.90$)$ \\
$2.52(2.14$ to 0.11$)$ \\
$7.5(6.8$ to 8.7$)$ \\
$0.36(0.24$ to 0.69$)$ \\
$6.8(6.0$ to 7.9$)$ \\
$1.3(0.5$ to 2.8$)$ \\
$0.6(0.3$ to 1.1$)$ \\
$2592.3(1560.8$ to 3021.3$)$ \\
$2568.9(2153.9$ to 3024.0$)$ \\
$0.12(-0.20$ to 0.55$)$ \\
$58.1(51.8$ to 69.0$)$ \\
$782.6(628.6$ to 1090.2$)$ \\
$104.6(68.5$ to 184.9$)$ \\
$0.14(-0.27$ to 0.58$)$ \\
$1.2(0.9$ to 1.4$)$ \\
$2.1(1.8$ to 2.3$)$ \\
\end{tabular}

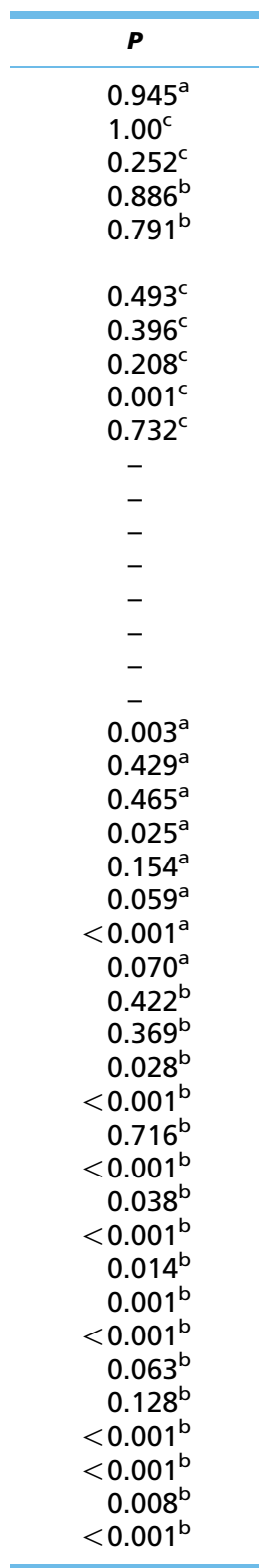

Data are given as mean (s.D.) (differences between type 1 diabetes and control patients were analysed using unpaired $t$-tests (normal distribution) ${ }^{a}$, median (interquartile range) (differences between type 1 diabetes and control patients were analysed using Mann-Whitney $U$ tests (data not-normally distributed) $)^{b}$, or percentages (differences between patients with type 1 diabetes and control patients were analysed using the $\chi^{2}$-test) ${ }^{c}$. CHD, coronary heart disease; T2DM, type 2 diabetes; T1DM, type 1 diabetes; WHR, waist-to-hip ratio; ACR, urinary albumin/creatinine ratio.

http://joe.endocrinology-journals.org DOI: 10.1530/JOE-13-0407
๑) 2014 Society for Endocrinology Printed in Great Britain
Published by Bioscientifica Ltd. 


\section{Results}

The main clinical and analytical characteristics of the studied population are given in Table 1. Regarding pharmacological medications, eight subjects were being treated with antihypertensive drugs (seven with type 1 diabetes), 15 with statins (14 patients) and six with antiplatelet drugs (all patients). Subjects with type 1 diabetes, compared with their non-diabetic counterparts, were more hypertensive and had higher values of BMI, fasting plasma glucose, and HbA1c and higher concentrations of inflammatory-related serum proteins. In addition, subjects with type 1 diabetes presented lower concentrations of LDL-cholesterol, probably due to the great number of patients treated with statins, or differences in dietary habits.

\section{Association between circulating levels of CML and skin autofluorescence with clinical and analytical variables:}

Subjects with type 1 diabetes had higher serum concentrations of CML (1.18 $\mu \mathrm{g} / \mathrm{ml}(0.94-1.40)$ vs $0.96 \mu \mathrm{g} / \mathrm{ml}(0.77-1.23)$; $P=0.008)$ and higher levels of skin autofluorescence $(2.10$ (1.80-2.30) vs 1.70 (1.60-2.10); $P<0.001)$, when compared with their respective controls (Table 1$)$. These differences remained significant after adjustment for classical cardiovascular risk factors (age, gender, physical activity, smoking, hypertension, dyslipidaemia and BMI) using multiple linear regression analysis $(\beta=0.280 ; P=0.023$ and $\beta=0.341$; $P<0.001$ for CML and skin autofluorescence respectively).
Tables 2 and 3 show univariate correlations between CML and skin autofluorescence with cardiovascular risk factors in the whole population: healthy subjects and patients with type 1 diabetes respectively. In the whole population, CML was associated with the presence of dyslipidaemia, type 1 diabetes, fasting plasma glucose and HbA1c (Table 2). Skin autofluorescence was associated with age, smoking status, the presence of hypertension and dyslipidaemia, BMI, type 1 diabetes, fasting plasma glucose and HbA1c (Table 3). In healthy subjects, CML was correlated only with the presence of dyslipidaemia (Table 2), while skin autofluorescence was positively correlated with age, total and LDL-cholesterol, and triglycerides (Table 3). In type 1 diabetes, skin autofluorescence was positively associated with age, BMI and diabetes duration $(r=0.291 ; P=0.021$; Table 3$)$. However, no association was observed between serum concentrations of CML and cardiovascular risk factors or glycaemic control (Table 2) in these patients. No relationship was found between serum concentrations of CML and skin autofluorescence $(r=0,054$; $P=0.586$ for the whole population, $r=0.053 ; P=0.736$ for the healthy subjects and $r=0.114 ; P=0.374$ for subjects with type 1 diabetes).

\section{Relationship between aPWV and AGE activity}

Patients with type 1 diabetes had higher aPWV compared with their respective controls (6.8 (6.0-7.9) vs 6.1 (5.5-6.7); $P<0.001)$. Skin autofluorescence was positively associated with aPWV only in type 1 diabetes

Table 2 Spearman correlation coefficients for the association between CML and classical cardiovascular risk factors. Data are given as Spearman correlation coefficients ( $P$ value)

Age (years)
Gender (female)
Diabetes mellitus (N/Y)
Smoking (N/Y)
Physical activity (METS, min/week)
Hypertension (N/Y)
Dyslipidaemia
BMI (kg/m²)
Waist $(\mathrm{cm})$
WHR
SBP (mmHg)
DBP (mmHg)
MAP (mmHg)
Total cholesterol (mmol/l)
Triglycerides (mmol/l)
HDL-cholesterol (mmol/l)
LDL-cholesterol (mmol/l)
FPG (mmol/l)
HbA1c (\%)

All
$-0.092(0.288)$
$0.109(0.206)$
$0.227(0.008)$
$0.025(0.770)$
$-0.111(0.202)$
$-0.147(0.087)$
$0.221(0.010)$
$-0.008(0.922)$
$-0.107(0.216)$
$-0.063(0.466)$
$-0.085(0.326)$
$-0.121(0.160)$
$-0.124(0.150)$
$0.095(0.270)$
$0.052(0.547)$
$0.044(0.609)$
$0.055(0.524)$
$0.189(0.027)$
$0.279(0.001)$

\begin{tabular}{r}
\hline Healthy subjects \\
$-0.019(0.875)$ \\
$0.135(0.273)$ \\
- \\
$0.174(0.155)$ \\
$-0.085(0.492)$ \\
$-0.066(0.595)$ \\
$0.255(0.036)$ \\
$-0.145(0.237)$ \\
$-0.209(0.088)$ \\
$-0.022(0.859)$ \\
$0.093(0.452)$ \\
$-0.049(0.693)$ \\
$-0.006(0.985)$ \\
$0.120(0.331)$ \\
$0.031(0.800)$ \\
$0.032(0.798)$ \\
$0.089(0.470)$ \\
$0.042(0.735)$ \\
$0.152(0.215)$ \\
\end{tabular}

\begin{tabular}{c}
\hline Type 1 diabetes \\
$-0.178(0.146)$ \\
$0.073(0.552)$ \\
- \\
$-0.178(0.147)$ \\
$-0.194(0.118)$ \\
$-0.207(0.116)$ \\
$0.208(0.089)$ \\
$-0.033(0.791)$ \\
$-0.047(0.701)$ \\
$-0.141(0.251)$ \\
$-0.189(0.153)$ \\
$-0.169(0.202)$ \\
$-0.227(0.084)$ \\
$0.121(0.327)$ \\
$0.066(0.593)$ \\
$0.025(0.838)$ \\
$0.087(0.483)$ \\
$0.041(0.739)$ \\
$0.151(0.219)$ \\
\end{tabular}

SBP, systolic blood pressure; DBP, diastolic blood pressure; MAP, mean arterial pressure; FPG, fasting plasma glucose.

http://joe.endocrinology-journals.org DOI: 10.1530/JOE-13-0407
(C) 2014 Society for Endocrinology Printed in Great Britain
Published by Bioscientifica Ltd. 
Table 3 Spearman correlation coefficients for the association between skin autofluorescence and classical cardiovascular risk factors. Data are given as Spearman correlation coefficients ( $P$ value)

Age (years)
Gender (female)
Diabetes mellitus (N/Y)
Smoking (N/Y)
Physical activity (METS-min/week)
Hypertension (N/Y)
Dyslipidaemia
BMI (kg/m²)
Waist (cm)
WHR
SBP (mmHg)
DBP (mmHg)
MAP (mmHg)
Total cholesterol (mmol/l)
Triglycerides (mmol/l)
HDL-cholesterol (mmol/l)
LDL-cholesterol (mmol/l)
FPG (mmol/l)
HbA1c (\%)

\begin{tabular}{l}
\multicolumn{1}{c}{ All } \\
\hline $0.444(<0.001)$ \\
$0.178(0.067)$ \\
$0.390(<0.001)$ \\
$0.362(<0.001)$ \\
$-0.160(0.104)$ \\
$0.203(0.721)$ \\
$0.203(0.037)$ \\
$0.274(0.005)$ \\
$0.222(0.022)$ \\
$0.116(0.235)$ \\
$0.108(0.269)$ \\
$0.123(0.209)$ \\
$0.138(0.159)$ \\
$0.175(0.072)$ \\
$0.107(0.273)$ \\
$0.094(0.337)$ \\
$0.151(0.123)$ \\
$0.305(0.001)$ \\
$0.407(<0.001)$
\end{tabular}

\begin{tabular}{c}
\hline Healthy subjects \\
\hline $0.499(0.001)$ \\
$0.199(0.201)$ \\
- \\
$0.340(0.026)$ \\
$-0.124(0.428)$ \\
$0.120(0.445)$ \\
$0.247(0.111)$ \\
$0.170(0.275)$ \\
$0.095(0.545)$ \\
$0.034(0.828)$ \\
$0.041(0.796)$ \\
$0.041(0.792)$ \\
$0.077(0.255)$ \\
$0.467(0.002)$ \\
$0.343(0.024)$ \\
$0.034(0.830)$ \\
$0.492(0.001)$ \\
$0.019(0.905)$ \\
$0.133(0.396)$
\end{tabular}

\begin{tabular}{c}
\hline Type 1 diabetes \\
\hline $0.494(<0.001)$ \\
$0.221(0.082)$ \\
- \\
$0.241(0.057)$ \\
$-0.230(0.074)$ \\
$0.098(0.445)$ \\
$0.184(0.149)$ \\
$0.258(0.041)$ \\
$0.292(0.020)$ \\
$0.142(0.266)$ \\
$0.035(0.784)$ \\
$0.014(0.911)$ \\
$0.046(0.721)$ \\
$0.126(0.324)$ \\
$-0.057(0.656)$ \\
$0.162(0.204)$ \\
$0.097(0.449)$ \\
$0.049(0.704)$ \\
$0.193(0.129)$
\end{tabular}

SBP, systolic blood pressure; DBP, diastolic blood pressure; MAP, mean arterial pressure; FPG, fasting plasma glucose.

patients $(r=0.370 ; P=0.003)$ and not for the control group $(r=0.186 ; P=0.233)$. No association between CML and aPWV was found (type 1 diabetes: $r=-0.034 ; P=0.781$ and healthy subjects: $r=-0.139 ; \mathrm{p}=0.259)$.

In order to strengthen the identification of the observed associations, we constructed a multiple linear regression analysis, considering aPWV as the dependent variable and adjusting for classical cardiovascular risk factors. We found that skin autofluorescence was the strongest factor associated with aPWV in type 1 diabetes $(\beta=0.380 ; P<0.001$; Table 4). Therefore, skin autofluorescence contributed to $14 \%$ of aPWV variance. Additional adjustments for HbA1c, disease duration, low-grade inflammation and endothelial dysfunction did not change these results.

\section{Discussion}

Non-invasive skin intrinsic fluorescence using the fluorescent properties of certain collagen AGEs is gaining acceptance as a novel marker of accumulation of AGEs. In this work, we show for the first time, to our knowledge, that skin autofluorescence in patients with type 1 diabetes (a high cardiovascular risk population) is one of the main factors associated with the increase of AS, determined by an accurate index such as aPWV, before development of cardiovascular disease.

AGEs are involved in the aetiology of micro- and macrovascular complications in diabetes mellitus (Wautier et al. 2003, Genuth et al. 2005, Nin et al. 2011). Additionally, skin autofluorescence is associated with the presence of cardiovascular disease in both type 1 and type 2 diabetes (Meerwaldt et al. 2007, Lutgers et al. 2009). Strikingly, serum levels of AGEs were predictive of cardiovascular events and all-cause mortality in a type 1 diabetes cohort (Nin et al. 2011). Our results are in agreement with these findings, and indicate that increased accumulation of tissue AGEs could be involved even in early stages of arteriosclerotic disease. Interestingly, skin

Table 4 Association between skin autofluorescence and aPWV in subjects with type 1 diabetes. Variables adjusted into this model were: age, gender, smoking, physical activity, hypertension (N/Y), dyslipidaemia (N/Y), BMI, mean arterial blood pressure, total cholesterol, log triglycerides, logHDL-cholesterol and skin autofluorescence. Only significant variables are shown in the table

\begin{tabular}{lcccc}
\hline log aPWV $\left(R=0.737 ; R^{2}=0.543\right)$ & $B$ & s.D. \\
\cline { 1 - 1 } & & 0.007 & & 0.002 \\
MAP & & 0.003 & & 0.001 \\
Total cholesterol & & 0.001 & & 0.000 \\
Skin autofluorescence & & 0.370 & & 0.097
\end{tabular}

http://joe.endocrinology-journals.org DOI: 10.1530/JOE-13-0407
(C) 2014 Society for Endocrinology Printed in Great Britain

\begin{tabular}{c}
$\beta$ \\
\hline 0.338 \\
0.361 \\
0.287 \\
0.380 \\
\hline
\end{tabular}

\begin{tabular}{c}
$95 \% \mathbf{C l}$ \\
\hline $0.003-0.011$ \\
$0.001-0.004$ \\
$0.000-0.001$ \\
$0.175-0.565$ \\
\hline
\end{tabular}

$\begin{array}{r}\multicolumn{1}{c}{\boldsymbol{P}} \\ \hline 0.001 \\ 0.001 \\ 0.006 \\ <0.001 \\ \hline\end{array}$

\begin{tabular}{c}
$\boldsymbol{R}^{\mathbf{2}}$ change \\
0.117 \\
0.027 \\
0.036 \\
0.042 \\
\hline
\end{tabular}


autofluorescence remained associated with AS in type 1 diabetes after adjustment for classical cardiovascular risk factors, glycaemic control, disease duration, low-grade inflammation and endothelial dysfunction. Notably, this association was specific for type 1 diabetic patients because it was not detected in healthy subjects. Although there is general agreement about age as an important determinant of both AS and AGEs, in the type 1 diabetic population we failed to find this. Probably the narrow range of age of patients included in this study hinders the detection of evidence of this association in young subjects. Interestingly, this observation reinforces the importance of obtaining a powerful and sensitive marker of arterial derangement before clinical events appear. In this sense, we are tempted to propose quantification of skin AGEs as a simple and useful tool to detect high-cardiovascular-risk subjects in early stages of type 1 diabetes. Along these lines, Conway et al. (2012) recently described an association between skin autofluorescence and coronary artery calcification, another measure of pre-clinical arteriosclerosis. In addition, Januszewski et al. (2012) showed that skin autofluorescence was correlated with the measures of systemic AS assessed as pulse wave analysis, supporting our observations.

We are aware of the descriptive results of our study, and the difficulty in inferring mechanistic hypotheses in these patients. Indeed, there are two primary mechanisms by which the accumulation of AGEs can contribute to the development of AS (Zieman \& Kass 2004). On the one hand, AGEs can cause cross linking of collagen and elastin fibrils, which may lead to the loss of collagen elasticity and a subsequent reduction in arterial distensibility properties, resulting in increased AS (Sell \& Monnier 2012). On the other hand, RAGE triggers multiple signalling cascades, resulting in the activation of nuclear transcription factors and the secretion of pro-inflammatory cytokines and vascular adhesion molecules (Goldin et al. 2006). In contrast to skin autofluorescence, CML, a non-crosslinking AGE and a ligand of RAGE, was not associated with aPWV in our study. Therefore, the lack of association between CML and AS, and the fact that the association between AS and skin autofluorescence was independent of low-grade inflammation and endothelial dysfunction markers lead us to propose the hypothesis that the effect of AGEs on AS may be mainly induced by cross linking rather than activation of the AGE-RAGE axis. However, the data in the literature are confusing on this point. Thus, some AGE-crosslink breakers, such as ALT711, have been shown to decrease AS (Kass et al. 2001), while other studies have shown an association between non-crosslinking AGEs, such as CML, and AS (Schram et al. 2005). Thus, new studies are required before definite conclusions concerning the specific role of the different types of AGEs in the vascular pathology of type 1 diabetes can be reached. Differences in the degree of protein modification by accumulation of AGEs, rather than on the specific AGEs per se, may be an alternative explanation for these discrepancies.

We are aware that the major limitation of our study is its cross-sectional design, which makes it impossible to determine the predictive value of skin autofluorescence, and undoubtedly additional prospective studies are needed. As we have stated, although patients were recruited together with age- and sex-matched controls, we performed the analyses using an independent $t$-test. We chose this strategy because unpaired analyses were statistically more conservative. Thus, the lack of significant differences between the groups might be either because there was no difference between them, or because there was a difference but the statistical analysis chosen did not detect it. There are some technical limitations in determination of skin autofluorescence. Not all AGEs exhibit fluorescent properties and other tissue components that fluoresce in the same range might be confounders. However, the method has been validated against specific AGE levels in skin biopsies in healthy subjects as well as in patients with diabetes (Meerwaldt et al. 2004). In addition, fluorescence is a group reactivity, and does not allow for the collection of quantitative information on the individual compounds responsible for it. In addition, we measured only one type of circulating AGE and it was measured only once. This might have underestimated the association between AGEs and AS. In relation to glycemic control, other studies have found that skin AF correlated with mean Hb1Ac values ( 5 and 10 years previously) but not with the latest value (Genevieve et al. 2013). In our study, we only analysed the latest HbA1c value; probably this accounts for the lack of association between glycemic control and skin AF. Nevertheless, we believe that these criticisms do not reduce the relevance of the association reported in our study.

In conclusion, circulating AGEs, measured as CML plasma levels, and skin accumulation of AGEs, are increased in patients with type 1 diabetes without clinical cardiovascular disease. Skin autofluorescence is associated with AS independently of classical cardiovascular risk factors, glycaemic control, disease duration and low-grade inflammation. These findings indicate that the

Published by Bioscientifica Ltd. 
determination of tissue AGE accumulation may be a useful marker for AS in type 1 diabetes.

\section{Declaration of interest}

The authors declare that there is no conflict of interest that could be perceived as prejudicing the impartiality of the research reported.

\section{Funding}

Financial support was provided by i) the Fundació la Marató de TV3-2008 (Project No.081410), ii) FIS PS09/01360. Instituto de Salud Carlos III, Ministerio de Sanidad y Consumo, Spain, iii) Beca Taulí Recerca 2010 (Project No. 2011517), iv) an Intensification Grant to José Miguel GonzálezClemente (Instituto de Salud Carlos III. Ministerio de Sanidad y Consumo, Spain) and v) a Rio Hortega Research Fellowship to Gemma Llauradó (CM12/00044. Instituto de Salud Carlos III. Ministerio de Sanidad y Consumo, Spain). These financial sponsors were not involved in study design, data collection and analysis or manuscript production.

\section{Author contribution statement}

The authors have made the following declarations about their contributions: $\mathrm{G} L$ researched data, wrote the manuscript, contributed to the discussion and reviewed/edited the manuscript; V C-M researched data; C V contributed to the discussion and reviewed/edited the manuscript; $\mathrm{R} S$ contributed to the discussion and reviewed/edited the manuscript; $\mathrm{P}$ G researched data; A C researched data; J V contributed to the discussion and reviewed/edited the manuscript; J-M G-C wrote the manuscript, contributed to the discussion and reviewed/edited the manuscript.

\section{References}

Cavalcante JL, Lima JA, Redheuil A \& Al-Mallah MH 2011 Aortic stiffness: current understanding and future directions. Journal of the American College of Cardiology 57 1511-1522. (doi:10.1016/j.jacc.2010.12.017)

Choi KM, Yoo HJ, Kim HY, Lee KW, Seo JA, Kim SG, Kim NH, Choi DS \& Baik SH 2009 Association between endogenous secretory RAGE, inflammatory markers and arterial stiffness. International Journal of Cardiology 132 96-101. (doi:10.1016/j.ijcard.2007.10.047)

Conway BN, Aroda VR, Maynard JD, Matter N, Fernandez S, Ratner RE \& Orchard TJ 2012 Skin intrinsic fluorescence is associated with coronary artery disease in individuals with long duration of type 1 diabetes. Diabetes Care 35 2331-2336. (doi:10.2337/dc12-0053)

Genevieve M, Vivot A, Gonzalez C, Raffaitin C, Barberger-Gateau P, Gin H \& Rigalleau V 2013 Skin autofluorescence is associated with past glycaemic control and complications in type 1 diabetes mellitus. Diabetes \& Metabolism 39 349-354. (doi:10.1016/j.diabet.2013.03.003)

Genuth S, Sun W, Cleary P, Sell DR, Dahms W, Malone J, Sivitz W \& Monnier VM 2005 Glycation and carboxymethyllysine levels in skin collagen predict the risk of future 10-year progression of diabetic retinopathy and nephropathy in the diabetes control and complications trial and epidemiology of diabetes interventions and complications participants with type 1 diabetes. Diabetes 54 3103-3111. (doi:10.2337/diabetes.54.11.3103)

Goldin A, Beckman JA, Schmidt AM \& Creager MA 2006 Advanced glycation end products: sparking the development of diabetic vascular injury. Circulation 114 597-605. (doi:10.1161/CIRCULATION AHA.106.621854)
Hallal PC \& Victora CG 2004 Reliability and validity of the International Physical Activity Questionnaire (IPAQ). Medicine and Science in Sports and Exercise 36 556. (doi:10.1249/01.MSS.0000117161.66394.07)

Januszewski AS, Sachithanandan N, Karschimkus C, O'Neal DN, Yeung CK, Alkatib N \& Jenkins AJ 2012 Non-invasive measures of tissue autofluorescence are increased in type 1 diabetes complications and correlate with a non-invasive measure of vascular dysfunction. Diabetic Medicine 29 726-733. (doi:10.1111/j.1464-5491.2011.03562.x)

Kass DA, Shapiro EP, Kawaguchi M, Capriotti AR, Scuteri A, deGroof RC \& Lakatta EG 2001 Improved arterial compliance by a novel advanced glycation end-product crosslink breaker. Circulation 104 1464-1470. (doi:10.1161/hc3801.097806)

Komosinska-Vassev K, Olczyk P, Winsz-Szczotka K, Klimek K \& Olczyk K 2012 Plasma biomarkers of oxidative and AGE-mediated damage of proteins and glycosaminoglycans during healthy ageing: a possible association with ECM metabolism. Mechanisms of Ageing and Development 133 538-548. (doi:10.1016/j.mad.2012.07.001)

Laurent S, Cockcroft J, Van Bortel L, Boutouyrie P, Giannattasio C, Hayoz D, Pannier B, Vlachopoulos C, Wilkinson I \& Struijker-Boudier H 2006 Expert consensus document on arterial stiffness: methodological issues and clinical applications. European Heart Journal 27 2588-2605. (doi:10.1093/eurheartj/ehl254)

Libby P, Nathan DM, Abraham K, Brunzell JD, Fradkin JE, Haffner SM, Hsueh W, Rewers M, Roberts BT, Savage PJ et al. 2005 Report of the National Heart, Lung, and Blood Institute-National Institute of Diabetes and Digestive and Kidney Diseases Working Group on Cardiovascular Complications of Type 1 Diabetes Mellitus. Circulation 111 3489-3493. (doi:10.1161/CIRCULATIONAHA.104.529651)

Llaurado G, Ceperuelo-Mallafre V, Vilardell C, Simo R, Freixenet N, Vendrell J \& Gonzalez-Clemente JM 2012 Arterial stiffness is increased in patients with type 1 diabetes without cardiovascular disease: a potential role of low-grade inflammation. Diabetes Care 35 1083-1089. (doi:10.2337/dc11-1475)

Lutgers HL, Gerrits EG, Graaff R, Links TP, Sluiter WJ, Gans RO, Bilo HJ \& Smit AJ 2009 Skin autofluorescence provides additional information to the UK Prospective Diabetes Study (UKPDS) risk score for the estimation of cardiovascular prognosis in type 2 diabetes mellitus. Diabetologia 52 789-797. (doi:10.1007/s00125-009-1308-9)

Mancia G, De Backer G, Dominiczak A, Cifkova R, Fagard R, Germano G, Grassi G, Heagerty AM, Kjeldsen SE, Laurent S et al. 20072007 Guidelines for the management of arterial hypertension: The Task Force for the Management of Arterial Hypertension of the European Society of Hypertension (ESH) and of the European Society of Cardiology (ESC). European Heart Journal 28 1462-1536. (doi:10.1093/ eurheartj/ehm236)

McNulty M, Mahmud A \& Feely J 2007 Advanced glycation end-products and arterial stiffness in hypertension. American Journal of Hypertension 20 242-247. (doi:10.1016/j.amjhyper.2006.08.009)

Meerwaldt R, Graaff R, Oomen PH, Links TP, Jager JJ, Alderson NL, Thorpe SR, Baynes JW, Gans RO \& Smit AJ 2004 Simple non-invasive assessment of advanced glycation endproduct accumulation. Diabetologia 47 1324-1330. (doi:10.1007/s00125-004-1451-2)

Meerwaldt R, Lutgers HL, Links TP, Graaff R, Baynes JW, Gans RO \& Smit AJ 2007 Skin autofluorescence is a strong predictor of cardiac mortality in diabetes. Diabetes Care 30 107-112. (doi:10.2337/dc06-1391)

National Cholesterol Education Program (NCEP) Expert Panel on Detection, Evaluation, and Treatment of High Blood Cholesterol in Adults (Adult Treatment Panel III) 2002 Third Report of the National Cholesterol Education Program (NCEP) Expert Panel on Detection, Evaluation, and Treatment of High Blood Cholesterol in Adults (Adult Treatment Panel III) final report. Circulation 106 3143-3421.

Nin JW, Jorsal A, Ferreira I, Schalkwijk CG, Prins MH, Parving HH, Tarnow L, Rossing P \& Stehouwer CD 2011 Higher plasma levels of advanced glycation end products are associated with incident cardiovascular disease and all-cause mortality in type 1 diabetes: a 12-year follow-up study. Diabetes Care 34 442-447. (doi:10.2337/dc10-1087) 
Schram MT, Schalkwijk CG, Bootsma AH, Fuller JH, Chaturvedi N \& Stehouwer CD 2005 Advanced glycation end products are associated with pulse pressure in type 1 diabetes: the EURODIAB Prospective Complications Study. Hypertension 46 232-237. (doi:10.1161/01.HYP. 0000164574.60279.ba)

Sell DR \& Monnier VM 2012 Molecular basis of arterial stiffening: role of glycation. Gerontology $\mathbf{5 8}$ 227-237. (doi:10.1159/000334668)

Semba RD, Najjar SS, Sun K, Lakatta EG \& Ferrucci L 2009 Serum carboxymethyl-lysine, an advanced glycation end product, is associated with increased aortic pulse wave velocity in adults. American Journal of Hypertension 22 74-79. (doi:10.1038/ajh. 2008.320)

Singh R, Barden A, Mori T \& Beilin L 2001 Advanced glycation end-products: a review. Diabetologia 44 129-146. (doi:10.1007/ s001250051591)

Ueno H, Koyama H, Tanaka S, Fukumoto S, Shinohara K, Shoji T, Emoto M, Tahara H, Kakiya R, Tabata T et al. 2008 Skin autofluorescence, a marker for advanced glycation end product accumulation, is associated with arterial stiffness in patients with end-stage renal disease. Metabolism: Clinical and Experimental 57 1452-1457. (doi:10.1016/j.metabol.2008.05.016)

Vlachopoulos C, Aznaouridis K \& Stefanadis C 2010 Prediction of cardiovascular events and all-cause mortality with arterial stiffness: a systematic review and meta-analysis. Journal of the American College of Cardiology 55 1318-1327. (doi:10.1016/j.jacc.2009.10.061)

Wautier MP, Massin P, Guillausseau PJ, Huijberts M, Levy B, Boulanger E, Laloi-Michelin M \& Wautier JL 2003 N(carboxymethyl)lysine as a biomarker for microvascular complications in type 2 'diabetic patients. Diabetes \& Metabolism 29 44-52. (doi:10.1016/S12623636(07)70006-X)

Zieman SJ \& Kass DA 2004 Advanced glycation endproduct crosslinking in the cardiovascular system: potential therapeutic target for cardiovascular disease. Drugs 64 459-470. (doi:10.2165/00003495200464050-00001)

Zieman SJ, Melenovsky V \& Kass DA 2005 Mechanisms, pathophysiology, and therapy of arterial stiffness. Arteriosclerosis, Thrombosis, and Vascular Biology 25 932-943. (doi:10.1161/01.ATV.0000160548.78317.29)

Received in final form 19 March 2014

Accepted 28 March 2014

Accepted Preprint published online 28 March 2014 http://joe.endocrinology-journals.org DOI: 10.1530/JOE-13-0407
(C) 2014 Society for Endocrinology Printed in Great Britain
Published by Bioscientifica Ltd. 